Acta Crystallographica Section E

\section{Structure Reports}

Online

ISSN 1600-5368

Gregory S. Coumbarides, ${ }^{a}$ Marco Dingjan, a Jason Eames, ${ }^{b_{*}}$ Majid Motevalli ${ }^{a}$ and Nela Malatesti ${ }^{c}$

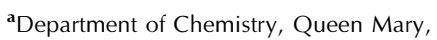
University of London, Mile End Road, London E1 4NS, England, 'bepartment of Chemistry, University of Hull, Cottingham Road, Kingstonupon-Hull HU6 7RX, England, and ${ }^{\mathbf{c}}$ Department of Chemistry, J. J. Strossmayer University of Osijek, Trg Sv. Trojstva 3, Osijek 31000, Croatia

Correspondence e-mail: j.eames@hull.ac.uk

\section{Key indicators}

Single-crystal X-ray study

$T=160 \mathrm{~K}$

Mean $\sigma(\mathrm{C}-\mathrm{C})=0.005 \AA$

$R$ factor $=0.042$

$w R$ factor $=0.101$

Data-to-parameter ratio $=8.4$

For details of how these key indicators were automatically derived from the article, see http://journals.iucr.org/e.

\title{
(+)-(4R,5S)-3-[2(S)-(4-Isobutylphenyl)propionyl]- 4-methyl-5-phenyloxazolidin-2-one
}

In the title compound, $\mathrm{C}_{23} \mathrm{H}_{27} \mathrm{NO}_{3}$, formed from enantiomerically pure $(+)-(4 R, 5 S)-4-m e t h y l-5$-phenyl-2-oxazolidinone and racemic 2-(4-isobutylphenyl)propanoyl chloride, the two carbonyl groups are oriented anti to each other, and the methyl group of the (4-isobutylphenyl)propionyl substituent lies close to the mean plane of the five-membered ring.

\section{Comment}

The title compound, (I), is the second in a series of structurally related compounds, introduced in our previous report (Coumbarides et al., 2006). With $R^{1}=4-\left({ }^{i} \mathrm{Bu}\right) \mathrm{C}_{6} \mathrm{H}_{4}$, the reaction shown in that report yielded the anti-syn and syn-syn diastereomers in 34 and $32 \%$ yields, respectively. The title compound, (I), is the syn-syn diastereomer (Fig. 1). In the crystal structure, the conformation of the central portion of the molecule is closely comparable with that in the phenyl derivative (Coumbarides et al., 2006). The conformation of the five-membered ring is similar, with atoms C1 and C2 lying respectively 0.170 (6) $\AA$ above and 0.298 (6) $\AA$ below the plane defined by atoms $\mathrm{O} 1, \mathrm{O} 2, \mathrm{~N} 1$ and $\mathrm{C} 3$. The carbonyl groups $(\mathrm{C} 3=\mathrm{O} 2$ and $\mathrm{C} 11=\mathrm{O} 3)$ are oriented anti to each other, with the torsion angle $\mathrm{O} 3-\mathrm{C} 11-\mathrm{N} 1-\mathrm{C} 3=$ $-179.7(4)^{\circ}$. The principal difference between the conformations of (I) and the phenyl derivative lies in the orientation of the (4-isobutylphenyl)propionyl substituent with respect to the remainder of the molecule: in (I), the torsion angle $\mathrm{N} 1-$ $\mathrm{C} 11-\mathrm{C} 12-\mathrm{C} 13=-90.2(4)^{\circ}$ compared with $-166.97(16)^{\circ}$ for the comparable measurement in the phenyl derivative. Thus, the C19 methyl group lies closer to the plane of the fivemembered ring in (I), in contrast with the anti arrangement observed for the C4 and C19 methyl groups in the phenyl derivative.<smiles>CC(C)c1ccc([C@H](C)C(=O)N2C(=O)O[C@@H](P)[C@@H]2C)cc1</smiles>

(I)
Received 27 June 2006 Accepted 12 August 2006

\section{Experimental}

The experimental procedure is comparable with that reported previously (Coumbarides et al., 2006). The actual quantities used for preparation of (I) were: $n$-butyllithium $(0.22 \mathrm{ml}, 2.5 M$ in hexanes, $0.56 \mathrm{mmol})$ and $(R, S)$-oxazolidinone $(0.1 \mathrm{~g}, 0.56 \mathrm{mmol})$ in $10 \mathrm{ml}$ tetrahydrofuran (THF), combined with a solution of (rac)-2-(4- 


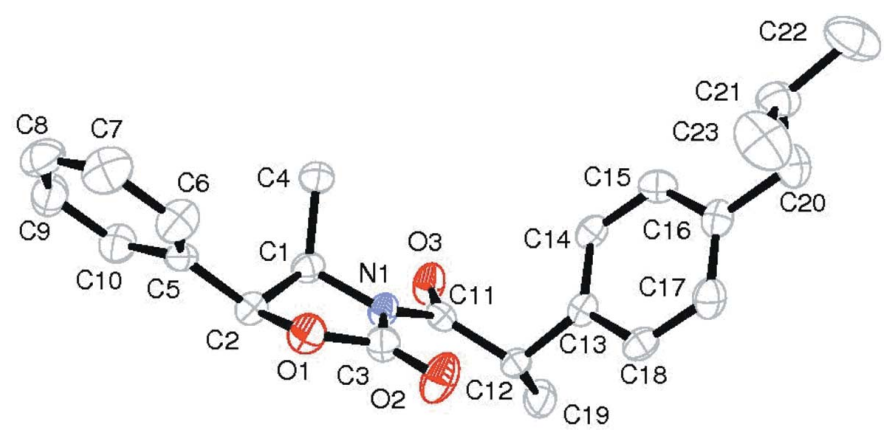

Figure 1

The molecular structure of the title compound, showing the atomlabelling scheme. Displacement ellipsoids are drawn at the $50 \%$ probability level and $\mathrm{H}$ atoms have been omitted.

isobutylphenyl)propanoyl chloride $(0.125 \mathrm{~g}, 0.56 \mathrm{mmol})$ in $1 \mathrm{ml}$ THF. The crude residue was purified by flash column chromatography on silica gel, eluting with light petroleum (b.p. 313-333 K)/diethyl ether (1:1) to give a separable diastereoisomeric mixture (in the ratio antisyn:syn-syn 54:46). The syn-syn diastereomer was obtained as colourless crystals $\left\{67 \mathrm{mg}, 32 \%\right.$ yield, m.p. 394-396 K, $R_{\mathrm{F}} 0.55$ [light petroleum (b.p 313-333 K)/diethyl ether, 1:1]\}. Spectroscopic analysis: $[\alpha]_{\mathrm{D}}^{25}=+98.1\left(\mathrm{CHCl}_{3}, 293 \mathrm{~K}\right.$, concentration $1.3 \mathrm{~g}$ per $\left.100 \mathrm{ml}\right)$; IR $\left(\mathrm{CHCl}_{3}, v_{\max }, \mathrm{cm}^{-1}\right): 1770(\mathrm{C}=\mathrm{O}), 1699(\mathrm{C}=\mathrm{O}) ;{ }^{1} \mathrm{H}$ NMR $\left(250 \mathrm{MHz} ; \mathrm{CDCl}_{3}\right): \delta 7.38-7.16(7 \mathrm{H}, m, 7 \times \mathrm{CH} ; \mathrm{Ar}$ and $\mathrm{Ph}), 7.08(2 \mathrm{H}$, $d, J=8.2 \mathrm{~Hz}, 2 \times \mathrm{CH}$; Ar), $5.63(1 \mathrm{H}, d, J=7.4 \mathrm{~Hz}, \mathrm{CHO}), 5.05(1 \mathrm{H}, q$, $J=7.1 \mathrm{~Hz}, \mathrm{ArCH}), 4.81(1 \mathrm{H}, m, \mathrm{CHN}), 2.43\left(2 \mathrm{H}, d, J=7.2 \mathrm{~Hz}, \mathrm{CH}_{2}\right)$, 1.89-1.79 (1H, $\left.\left.m, \mathrm{CH}\left(\mathrm{CH}_{3}\right)_{2}\right)\right), 1.48\left(3 \mathrm{H}, d, J=7.1 \mathrm{~Hz}, \mathrm{CH}_{3} \mathrm{CHAr}\right)$, $0.88\left(3 \mathrm{H}, d, J=6.7 \mathrm{~Hz}, \mathrm{CH}_{3}{ }^{a} \mathrm{CHCH}_{3}{ }^{b}\right), 0.87(3 \mathrm{H}, d, J=6.7 \mathrm{~Hz}$, $\left.\mathrm{CH}_{3}{ }^{a} \mathrm{CHCH}_{3}{ }^{b}\right), 0.72\left(3 \mathrm{H}, d, J=6.7 \mathrm{~Hz}, \mathrm{CH}_{3} \mathrm{CHN}\right) ;{ }^{13} \mathrm{C} \mathrm{NMR}$ $\left(100.6 \mathrm{MHz} ; \mathrm{CDCl}_{3}\right): \delta 174.6(\mathrm{NC}=\mathrm{O}), 152.6(\mathrm{OC}=\mathrm{O}), 140.5(i-\mathrm{C}$; Ar), 137.5 ( $i$-C; Ar), 133.6 ( $i$-C; Ph), 129.3, 127.7 ( $2 \times \mathrm{CH}$; Ar), 129.1, 128.7 and $125.8(3 \times \mathrm{CH} ; \mathrm{Ph}), 78.8(\mathrm{OCHPh}), 54.7(\mathrm{CHN}), 45.1$

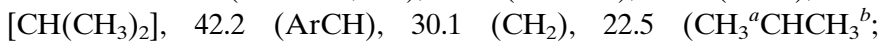
isobutylphenyl), $19.4\left(\mathrm{CH}_{3} \mathrm{CH}\right), 14.2\left(\mathrm{CH}_{3} \mathrm{CHN}\right)$; found: $M$ 365.1986; $\mathrm{C}_{23} \mathrm{H}_{27} \mathrm{NO}_{3}$ requires 365.1985.

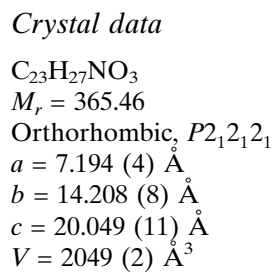

\section{Data collection}

Enraf-Nonius CAD-4 diffractometer $\omega / 2 \theta$ scans

Absorption correction: none

2141 measured reflections

2079 independent reflections

Refinement

Refinement on $F^{2}$

$R\left[F^{2}>2 \sigma\left(F^{2}\right)\right]=0.042$

$w R\left(F^{2}\right)=0.101$

$S=1.00$

2079 reflections

248 parameters

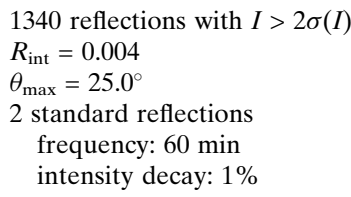

$\mathrm{H}$-atom parameters constrained $w=1 /\left[\sigma^{2}\left(F_{\mathrm{o}}{ }^{2}\right)+(0.0466 P)^{2}\right]$ where $P=\left(F_{\mathrm{o}}{ }^{2}+2 F_{\mathrm{c}}{ }^{2}\right) / 3$ $(\Delta / \sigma)_{\max }<0.001$ $\Delta \rho_{\max }=0.18 \mathrm{e} \AA^{-3}$ $\Delta \rho_{\min }=-0.20 \mathrm{e}^{-3}$

$\mathrm{H}$ atoms were placed in geometrically idealised positions and constrained to ride on their parent atoms, with $\mathrm{C}-\mathrm{H}=0.95-1.00 \AA$ and $U_{\text {iso }}(\mathrm{H})=1.2 U_{\text {eq }}(\mathrm{C})$ or $1.5 U_{\text {eq }}($ methyl $\mathrm{C})$. The methyl groups were allowed to rotate about their local threefold axes. In the absence of significant anomalous scattering effects, Friedel pairs have been merged. The absolute configuration is assigned on the basis of the known configuration of the starting material (Coumbarides et al., 2006).

Data collection: CAD-4 EXPRESS (Enraf-Nonius, 1994); cell refinement: CAD-4 EXPRESS; data reduction: XCAD4 (Harms \& Wocadlo, 1995); program(s) used to solve structure: SHELXS97 (Sheldrick, 1997); program(s) used to refine structure: SHELXL97 (Sheldrick, 1997); molecular graphics: ORTEP-3 for Windows (Farrugia, 1997); software used to prepare material for publication: Win $G X$ publication routines (Farrugia, 1999).

We are grateful to Onyx Scientific Limited (Drs Tony Flinn and Julian Northen) and Queen Mary, University of London for a studentship to MD, the Royal Society and the University of London Central Research Fund for financial support to JE, and the EPSRC National Mass Spectrometry Service (Swansea) for accurate mass determination.

\section{References}

Coumbarides, G. S., Eames, J., Motevalli, M., Malatesti, N. \& Yohannes, Y. (2006). Acta Cryst. E62, o4032-04034.

Enraf-Nonius (1994). CAD-4 EXPRESS. Enraf-Nonius, Delft, The Netherlands.

Farrugia, L. J. (1997). J. Appl. Cryst. 30, 565.

Farrugia, L. J. (1999). J. Appl. Cryst. 32, 837-838.

Harms, K. \& Wocadlo, S. (1995). XCAD4. University of Marburg, Germany.

Sheldrick, G. M. (1997). SHELXS97 and SHELXL97. University of Göttingen, Germany. 


\section{supporting information}

Acta Cryst. (2006). E62, o4035-o4036 [https://doi.org/10.1107/S1600536806031849]

(+)-(4R,5S)-3-[2(S)-(4-Isobutylphenyl) propionyl]- 4-methyl-5-phenyl-

oxazolidin-2-one

Gregory S. Coumbarides, Marco Dingjan, Jason Eames, Majid Motevalli and Nela Malatesti

(+)-(4R,5S)-3-[2(S)-(4-Isobutylphenyl)propionyl]- 4-methyl-5-phenyloxazolidin-2-one

\section{Crystal data}

$\mathrm{C}_{23} \mathrm{H}_{27} \mathrm{NO}_{3}$

$M_{r}=365.46$

Orthorhombic, $P 22_{1} 2_{1}$

Hall symbol: P 2ac 2ab

$a=7.194$ (4) $\AA$

$b=14.208(8) \AA$

$c=20.049(11) \AA$

$V=2049(2) \AA^{3}$

$Z=4$

Data collection

Enraf-Nonius CAD-4

diffractometer

Radiation source: Enraf Nonius FR590

Graphite monochromator

$\omega / 2 \theta$ scans

2141 measured reflections

2079 independent reflections

1340 reflections with $I>2 \sigma(I)$

\section{Refinement}

Refinement on $F^{2}$

Least-squares matrix: full

$R\left[F^{2}>2 \sigma\left(F^{2}\right)\right]=0.042$

$w R\left(F^{2}\right)=0.101$

$S=1.00$

2079 reflections

248 parameters

0 restraints

Primary atom site location: structure-invariant direct methods
$F(000)=784$

$D_{\mathrm{x}}=1.185 \mathrm{Mg} \mathrm{m}^{-3}$

Mo $K \alpha$ radiation, $\lambda=0.71073 \AA$

Cell parameters from 25 reflections

$\theta=10.0-12.3^{\circ}$

$\mu=0.08 \mathrm{~mm}^{-1}$

$T=160 \mathrm{~K}$

Block, colourless

$0.63 \times 0.38 \times 0.15 \mathrm{~mm}$

$R_{\text {int }}=0.004$

$\theta_{\max }=25.0^{\circ}, \theta_{\min }=1.8^{\circ}$

$h=0 \rightarrow 8$

$k=0 \rightarrow 16$

$l=0 \rightarrow 23$

2 standard reflections every $60 \mathrm{~min}$ intensity decay: $1 \%$

Secondary atom site location: difference Fourier map

Hydrogen site location: inferred from neighbouring sites

$\mathrm{H}$-atom parameters constrained

$w=1 /\left[\sigma^{2}\left(F_{\mathrm{o}}^{2}\right)+(0.0466 P)^{2}\right]$

where $P=\left(F_{\mathrm{o}}^{2}+2 F_{\mathrm{c}}^{2}\right) / 3$

$(\Delta / \sigma)_{\max }<0.001$

$\Delta \rho_{\max }=0.18 \mathrm{e} \AA^{-3}$

$\Delta \rho_{\min }=-0.20$ e $\AA^{-3}$

Absolute structure: assigned on the basis of known starting material 


\section{Special details}

Geometry. All e.s.d.'s (except the e.s.d. in the dihedral angle between two 1.s. planes) are estimated using the full covariance matrix. The cell e.s.d.'s are taken into account individually in the estimation of e.s.d.'s in distances, angles and torsion angles; correlations between e.s.d.'s in cell parameters are only used when they are defined by crystal symmetry.

An approximate (isotropic) treatment of cell e.s.d.'s is used for estimating e.s.d.'s involving 1.s. planes.

Least-squares planes $(x, y, z$ in crystal coordinates) and deviations from them (* indicates atom used to define plane) $0.2684(0.0131) x+8.7530(0.0193) y+15.7745(0.0224) z=7.3649(0.0082)$

$* 0.0000(0.0010) \mathrm{O} 1 * 0.0000(0.0013) \mathrm{O} 2 * 0.0000(0.0010) \mathrm{N} 1 * 0.0001(0.0033) \mathrm{C} 30.1698(0.0055) \mathrm{C} 1-0.2981$ $(0.0059) \mathrm{C} 2-1.3428(0.0082) \mathrm{C} 19$

Rms deviation of fitted atoms $=0.0001$

Refinement. Refinement of $F^{2}$ against ALL reflections. The weighted $R$-factor $w R$ and goodness of fit $S$ are based on $F^{2}$, conventional $R$-factors $R$ are based on $F$, with $F$ set to zero for negative $F^{2}$. The threshold expression of $F^{2}>2 \sigma\left(F^{2}\right)$ is used only for calculating $R$-factors(gt) $e t c$. and is not relevant to the choice of reflections for refinement. $R$-factors based on $F^{2}$ are statistically about twice as large as those based on $F$, and $R$ - factors based on ALL data will be even larger.

Fractional atomic coordinates and isotropic or equivalent isotropic displacement parameters $\left(\hat{A}^{2}\right)$

\begin{tabular}{|c|c|c|c|c|}
\hline & $x$ & $y$ & $z$ & $U_{\text {iso }} * / U_{\text {eq }}$ \\
\hline $\mathrm{C} 1$ & $0.2227(5)$ & $0.2446(2)$ & $0.33816(18)$ & $0.0277(9)$ \\
\hline H1 & 0.1236 & 0.2379 & 0.3035 & $0.033^{*}$ \\
\hline $\mathrm{C} 2$ & $0.2850(5)$ & $0.1467(2)$ & $0.36175(18)$ & $0.0289(9)$ \\
\hline $\mathrm{H} 2$ & 0.2635 & 0.1007 & 0.3248 & $0.035^{*}$ \\
\hline $\mathrm{C} 3$ & $0.5476(6)$ & $0.2262(3)$ & $0.3321(2)$ & $0.0339(10)$ \\
\hline $\mathrm{C} 4$ & $0.1573(5)$ & $0.3102(3)$ & $0.39234(18)$ & $0.0363(10)$ \\
\hline $\mathrm{H} 4 \mathrm{~A}$ & 0.2470 & 0.3099 & 0.4291 & $0.054^{*}$ \\
\hline H4B & 0.0358 & 0.2894 & 0.4087 & $0.054^{*}$ \\
\hline $\mathrm{H} 4 \mathrm{C}$ & 0.1465 & 0.3742 & 0.3744 & $0.054^{*}$ \\
\hline $\mathrm{C} 5$ & $0.1927(5)$ & 0.1101 & $0.42366(18)$ & $0.0296(9)$ \\
\hline C6 & $0.2798(6)$ & $0.1061(3)$ & $0.48455(17)$ & $0.0426(11)$ \\
\hline H6 & 0.4049 & 0.1266 & 0.4887 & $0.051^{*}$ \\
\hline $\mathrm{C} 7$ & $0.1862(7)$ & 0.0723 & $0.5399(2)$ & $0.0517(13)$ \\
\hline $\mathrm{H} 7$ & 0.2468 & 0.0705 & 0.5820 & $0.062 *$ \\
\hline $\mathrm{C} 8$ & $0.0071(6)$ & 0.0417 & $0.5342(2)$ & $0.0450(12)$ \\
\hline H8 & -0.0566 & 0.0179 & 0.5721 & $0.054^{*}$ \\
\hline C9 & $-0.0809(6)$ & $0.0453(3)$ & $0.4732(2)$ & $0.0446(11)$ \\
\hline H9 & -0.2062 & 0.0250 & 0.4692 & $0.053^{*}$ \\
\hline $\mathrm{C} 10$ & $0.0126(6)$ & 0.0783 & $0.4181(2)$ & $0.0373(11)$ \\
\hline H10 & -0.0476 & 0.0792 & 0.3759 & $0.045^{*}$ \\
\hline $\mathrm{C} 11$ & $0.3942(5)$ & $0.3446(2)$ & $0.25742(16)$ & $0.0267(8)$ \\
\hline $\mathrm{C} 12$ & $0.5717(5)$ & 0.3735 & $0.22302(17)$ & $0.0286(9)$ \\
\hline H12 & 0.6590 & 0.3187 & 0.2229 & $0.034^{*}$ \\
\hline C13 & $0.6625(5)$ & $0.4547(2)$ & $0.25974(18)$ & $0.0260(9)$ \\
\hline $\mathrm{C} 14$ & $0.5624(5)$ & 0.5283 & $0.28743(17)$ & $0.0286(9)$ \\
\hline H14 & 0.4305 & 0.5263 & 0.2866 & $0.034 *$ \\
\hline C15 & $0.6509(5)$ & $0.6051(3)$ & $0.31640(18)$ & $0.0322(9)$ \\
\hline H15 & 0.5786 & 0.6539 & 0.3358 & $0.039^{*}$ \\
\hline C16 & $0.8434(5)$ & $0.6115(3)$ & $0.31736(17)$ & $0.0308(9)$ \\
\hline C17 & $0.9411(5)$ & 0.5378 & 0.28901 (19) & $0.0362(10)$ \\
\hline $\mathrm{H} 17$ & 1.0730 & 0.5402 & 0.2888 & $0.043 *$ \\
\hline
\end{tabular}




$\begin{array}{lllll}\mathrm{C} 18 & 0.8537(5) & 0.4608(3) & 0.26105(18) & 0.0329(9) \\ \mathrm{H} 18 & 0.9262 & 0.4115 & 0.2425 & 0.039^{*} \\ \mathrm{C} 19 & 0.5285(6) & 0.4009(3) & 0.15034(17) & 0.0415(10) \\ \mathrm{H} 19 \mathrm{~A} & 0.4710 & 0.3473 & 0.1274 & 0.062^{*} \\ \mathrm{H} 19 \mathrm{~B} & 0.6441 & 0.4181 & 0.1276 & 0.062^{*} \\ \mathrm{H} 19 \mathrm{C} & 0.4429 & 0.4545 & 0.1498 & 0.062^{*} \\ \mathrm{C} 20 & 0.9382(6) & 0.6947(3) & 0.34867(18) & 0.0409(11) \\ \mathrm{H} 20 \mathrm{~A} & 0.8716 & 0.7526 & 0.3352 & 0.049^{*} \\ \mathrm{H} 20 \mathrm{~B} & 1.0664 & 0.6989 & 0.3310 & 0.049^{*} \\ \mathrm{C} 21 & 0.9471(6) & 0.6910(3) & 0.4245(2) & 0.0435(11) \\ \mathrm{H} 21 & 0.8188 & 0.6785 & 0.4415 & 0.052^{*} \\ \mathrm{C} 22 & 1.0109(7) & 0.7844(3) & 0.4524(2) & 0.0663(15) \\ \mathrm{H} 22 \mathrm{~A} & 1.0057 & 0.7825 & 0.5012 & 0.099^{*} \\ \mathrm{H} 22 \mathrm{~B} & 0.9295 & 0.8346 & 0.4359 & 0.099^{*} \\ \mathrm{H} 22 \mathrm{C} & 1.1389 & 0.7967 & 0.4380 & 0.099^{*} \\ \mathrm{C} 23 & 1.0722(7) & 0.6124(3) & 0.4485(2) & 0.0656(15) \\ \mathrm{H} 23 \mathrm{~A} & 1.2000 & 0.6246 & 0.4340 & 0.098^{*} \\ \mathrm{H} 23 \mathrm{~B} & 1.0297 & 0.5525 & 0.4297 & 0.098^{*} \\ \mathrm{H} 23 \mathrm{C} & 1.0678 & 0.6092 & 0.4973 & 0.098^{*} \\ \mathrm{~N} 1 & 0.3962(4) & 0.27652(19) & 0.30671(13) & 0.0269(7) \\ \mathrm{O} 1 & 0.4839(3) & 0.15713(18) & 0.37146(13) & 0.0376(7) \\ \text { O2 } & 0.7093(4) & 0.2401(2) & 0.32159(15) & 0.0477(8) \\ \text { O3 } & 0.2465(4) & 0.38040(17) & 0.24285(12) & 0.0381(7) \\ & & & & \end{array}$

Atomic displacement parameters $\left(\AA^{2}\right)$

\begin{tabular}{lllllll}
\hline & $U^{11}$ & $U^{22}$ & $U^{33}$ & $U^{12}$ & $U^{13}$ & $U^{23}$ \\
\hline C1 & $0.0224(19)$ & $0.032(2)$ & $0.0284(19)$ & $0.0008(18)$ & $0.0002(17)$ & $-0.0020(17)$ \\
C2 & $0.027(2)$ & $0.032(2)$ & $0.027(2)$ & $-0.0007(17)$ & $-0.0009(18)$ & $0.0006(17)$ \\
C3 & $0.034(3)$ & $0.033(2)$ & $0.035(2)$ & $-0.002(2)$ & $0.0005(19)$ & $0.003(2)$ \\
C4 & $0.036(2)$ & $0.032(2)$ & $0.041(2)$ & $0.0037(19)$ & $0.010(2)$ & $-0.0010(18)$ \\
C5 & $0.036(2)$ & $0.026(2)$ & $0.027(2)$ & $0.0018(19)$ & $0.0077(18)$ & $-0.0020(18)$ \\
C6 & $0.037(2)$ & $0.059(3)$ & $0.032(2)$ & $-0.005(2)$ & $-0.003(2)$ & $0.003(2)$ \\
C7 & $0.056(3)$ & $0.067(3)$ & $0.032(2)$ & $0.007(3)$ & $-0.006(2)$ & $0.009(2)$ \\
C8 & $0.051(3)$ & $0.050(3)$ & $0.033(2)$ & $0.008(3)$ & $0.010(2)$ & $0.007(2)$ \\
C9 & $0.042(3)$ & $0.048(3)$ & $0.044(2)$ & $-0.007(2)$ & $0.004(2)$ & $0.003(2)$ \\
C10 & $0.039(3)$ & $0.046(3)$ & $0.027(2)$ & $-0.005(2)$ & $-0.003(2)$ & $0.0012(19)$ \\
C11 & $0.029(2)$ & $0.0208(17)$ & $0.030(2)$ & $0.0013(18)$ & $-0.0017(19)$ & $-0.0009(17)$ \\
C12 & $0.023(2)$ & $0.030(2)$ & $0.033(2)$ & $0.0011(17)$ & $0.0050(17)$ & $-0.0012(17)$ \\
C13 & $0.021(2)$ & $0.030(2)$ & $0.027(2)$ & $0.0019(17)$ & $-0.0012(17)$ & $0.0052(19)$ \\
C14 & $0.021(2)$ & $0.036(2)$ & $0.029(2)$ & $0.0017(19)$ & $-0.0005(17)$ & $0.0073(19)$ \\
C15 & $0.034(2)$ & $0.033(2)$ & $0.029(2)$ & $0.009(2)$ & $-0.0019(19)$ & $-0.001(2)$ \\
C16 & $0.031(2)$ & $0.036(2)$ & $0.025(2)$ & $-0.001(2)$ & $-0.0042(18)$ & $0.007(2)$ \\
C17 & $0.023(2)$ & $0.041(2)$ & $0.045(3)$ & $-0.003(2)$ & $-0.0041(19)$ & $0.005(2)$ \\
C18 & $0.023(2)$ & $0.039(2)$ & $0.037(2)$ & $0.0060(19)$ & $0.0014(19)$ & $0.001(2)$ \\
C19 & $0.046(2)$ & $0.046(2)$ & $0.032(2)$ & $-0.015(2)$ & $0.003(2)$ & $-0.002(2)$ \\
C20 & $0.040(3)$ & $0.040(2)$ & $0.043(2)$ & $-0.006(2)$ & $-0.011(2)$ & $0.004(2)$ \\
C21 & $0.036(2)$ & $0.056(3)$ & $0.038(2)$ & $0.001(2)$ & $-0.007(2)$ & $-0.012(2)$
\end{tabular}




\begin{tabular}{lllllll}
\hline & & & & & \\
$\mathrm{C} 22$ & $0.061(3)$ & $0.066(3)$ & $0.072(3)$ & $0.007(3)$ & $-0.025(3)$ & $-0.032(3)$ \\
$\mathrm{C} 23$ & $0.084(4)$ & $0.060(3)$ & $0.053(3)$ & $-0.002(3)$ & $-0.032(3)$ & $0.005(3)$ \\
$\mathrm{N} 1$ & $0.0198(16)$ & $0.0300(17)$ & $0.0310(16)$ & $0.0022(14)$ & $0.0008(15)$ & $0.0051(14)$ \\
$\mathrm{O} 1$ & $0.0325(17)$ & $0.0388(15)$ & $0.0414(16)$ & $0.0045(13)$ & $-0.0004(13)$ & $0.0083(14)$ \\
$\mathrm{O} 2$ & $0.0227(16)$ & $0.061(2)$ & $0.0593(19)$ & $0.0015(14)$ & $0.0004(15)$ & $0.0207(16)$ \\
$\mathrm{O} 3$ & $0.0244(14)$ & $0.0374(14)$ & $0.0524(17)$ & $0.0030(14)$ & $-0.0007(14)$ & $0.0149(14)$ \\
\hline
\end{tabular}

Geometric parameters $\left(\AA,{ }^{\circ}\right)$

\begin{tabular}{|c|c|c|c|}
\hline $\mathrm{C} 1-\mathrm{N} 1$ & $1.470(4)$ & $\mathrm{C} 12-\mathrm{C} 19$ & $1.540(5)$ \\
\hline $\mathrm{C} 1-\mathrm{C} 4$ & $1.507(5)$ & $\mathrm{C} 12-\mathrm{H} 12$ & 1.000 \\
\hline $\mathrm{C} 1-\mathrm{C} 2$ & $1.536(5)$ & $\mathrm{C} 13-\mathrm{C} 18$ & $1.378(5)$ \\
\hline $\mathrm{C} 1-\mathrm{H} 1$ & 1.000 & $\mathrm{C} 13-\mathrm{C} 14$ & $1.386(5)$ \\
\hline $\mathrm{C} 2-\mathrm{O} 1$ & $1.452(4)$ & $\mathrm{C} 14-\mathrm{C} 15$ & $1.390(5)$ \\
\hline $\mathrm{C} 2-\mathrm{C} 5$ & $1.500(5)$ & C14-H14 & 0.950 \\
\hline $\mathrm{C} 2-\mathrm{H} 2$ & 1.000 & $\mathrm{C} 15-\mathrm{C} 16$ & $1.388(5)$ \\
\hline $\mathrm{C} 3-\mathrm{O} 2$ & $1.198(4)$ & $\mathrm{C} 15-\mathrm{H} 15$ & 0.950 \\
\hline $\mathrm{C} 3-\mathrm{O} 1$ & $1.340(4)$ & $\mathrm{C} 16-\mathrm{C} 17$ & $1.383(5)$ \\
\hline $\mathrm{C} 3-\mathrm{N} 1$ & $1.399(5)$ & $\mathrm{C} 16-\mathrm{C} 20$ & $1.502(5)$ \\
\hline $\mathrm{C} 4-\mathrm{H} 4 \mathrm{~A}$ & 0.980 & $\mathrm{C} 17-\mathrm{C} 18$ & $1.380(5)$ \\
\hline $\mathrm{C} 4-\mathrm{H} 4 \mathrm{~B}$ & 0.980 & C17-H17 & 0.950 \\
\hline $\mathrm{C} 4-\mathrm{H} 4 \mathrm{C}$ & 0.980 & C18-H18 & 0.950 \\
\hline $\mathrm{C} 5-\mathrm{C} 6$ & $1.373(5)$ & C19-H19A & 0.980 \\
\hline $\mathrm{C} 5-\mathrm{C} 10$ & $1.377(5)$ & C19-H19B & 0.980 \\
\hline $\mathrm{C} 6-\mathrm{C} 7$ & $1.384(5)$ & $\mathrm{C} 19-\mathrm{H} 19 \mathrm{C}$ & 0.980 \\
\hline $\mathrm{C} 6-\mathrm{H} 6$ & 0.950 & $\mathrm{C} 20-\mathrm{C} 21$ & $1.522(5)$ \\
\hline $\mathrm{C} 7-\mathrm{C} 8$ & $1.365(6)$ & $\mathrm{C} 20-\mathrm{H} 20 \mathrm{~A}$ & 0.990 \\
\hline $\mathrm{C} 7-\mathrm{H} 7$ & 0.950 & $\mathrm{C} 20-\mathrm{H} 20 \mathrm{~B}$ & 0.990 \\
\hline $\mathrm{C} 8-\mathrm{C} 9$ & $1.377(5)$ & $\mathrm{C} 21-\mathrm{C} 22$ & $1.512(6)$ \\
\hline $\mathrm{C} 8-\mathrm{H} 8$ & 0.950 & $\mathrm{C} 21-\mathrm{C} 23$ & $1.513(6)$ \\
\hline $\mathrm{C} 9-\mathrm{C} 10$ & $1.376(5)$ & $\mathrm{C} 21-\mathrm{H} 21$ & 1.000 \\
\hline C9- $\mathrm{H} 9$ & 0.950 & $\mathrm{C} 22-\mathrm{H} 22 \mathrm{~A}$ & 0.980 \\
\hline $\mathrm{C} 10-\mathrm{H} 10$ & 0.950 & $\mathrm{C} 22-\mathrm{H} 22 \mathrm{~B}$ & 0.980 \\
\hline $\mathrm{C} 11-\mathrm{O} 3$ & $1.214(4)$ & $\mathrm{C} 22-\mathrm{H} 22 \mathrm{C}$ & 0.980 \\
\hline $\mathrm{C} 11-\mathrm{N} 1$ & $1.383(4)$ & $\mathrm{C} 23-\mathrm{H} 23 \mathrm{~A}$ & 0.980 \\
\hline $\mathrm{C} 11-\mathrm{C} 12$ & $1.508(5)$ & $\mathrm{C} 23-\mathrm{H} 23 \mathrm{~B}$ & 0.980 \\
\hline $\mathrm{C} 12-\mathrm{C} 13$ & $1.517(5)$ & $\mathrm{C} 23-\mathrm{H} 23 \mathrm{C}$ & 0.980 \\
\hline $\mathrm{N} 1-\mathrm{C} 1-\mathrm{C} 4$ & $112.5(3)$ & $\mathrm{C} 14-\mathrm{C} 13-\mathrm{C} 12$ & $123.0(3)$ \\
\hline $\mathrm{N} 1-\mathrm{C} 1-\mathrm{C} 2$ & $99.5(3)$ & $\mathrm{C} 13-\mathrm{C} 14-\mathrm{C} 15$ & $121.5(3)$ \\
\hline $\mathrm{C} 4-\mathrm{C} 1-\mathrm{C} 2$ & $115.5(3)$ & $\mathrm{C} 13-\mathrm{C} 14-\mathrm{H} 14$ & 119.3 \\
\hline $\mathrm{N} 1-\mathrm{C} 1-\mathrm{H} 1$ & 109.7 & $\mathrm{C} 15-\mathrm{C} 14-\mathrm{H} 14$ & 119.3 \\
\hline $\mathrm{C} 4-\mathrm{C} 1-\mathrm{H} 1$ & 109.7 & $\mathrm{C} 16-\mathrm{C} 15-\mathrm{C} 14$ & 120.9 (4) \\
\hline $\mathrm{C} 2-\mathrm{C} 1-\mathrm{H} 1$ & 109.7 & $\mathrm{C} 16-\mathrm{C} 15-\mathrm{H} 15$ & 119.5 \\
\hline $\mathrm{O} 1-\mathrm{C} 2-\mathrm{C} 5$ & $111.1(3)$ & $\mathrm{C} 14-\mathrm{C} 15-\mathrm{H} 15$ & 119.5 \\
\hline $\mathrm{O} 1-\mathrm{C} 2-\mathrm{C} 1$ & $103.6(3)$ & $\mathrm{C} 17-\mathrm{C} 16-\mathrm{C} 15$ & $116.9(4)$ \\
\hline $\mathrm{C} 5-\mathrm{C} 2-\mathrm{C} 1$ & $116.1(3)$ & $\mathrm{C} 17-\mathrm{C} 16-\mathrm{C} 20$ & $122.5(3)$ \\
\hline $\mathrm{O} 1-\mathrm{C} 2-\mathrm{H} 2$ & 108.6 & $\mathrm{C} 15-\mathrm{C} 16-\mathrm{C} 20$ & $120.7(4)$ \\
\hline
\end{tabular}




\begin{tabular}{|c|c|c|c|}
\hline $\mathrm{C} 5-\mathrm{C} 2-\mathrm{H} 2$ & 108.6 & $\mathrm{C} 18-\mathrm{C} 17-\mathrm{C} 16$ & $122.3(4)$ \\
\hline $\mathrm{C} 1-\mathrm{C} 2-\mathrm{H} 2$ & 108.6 & $\mathrm{C} 18-\mathrm{C} 17-\mathrm{H} 17$ & 118.8 \\
\hline $\mathrm{O} 2-\mathrm{C} 3-\mathrm{O} 1$ & $123.8(4)$ & $\mathrm{C} 16-\mathrm{C} 17-\mathrm{H} 17$ & 118.8 \\
\hline $\mathrm{O} 2-\mathrm{C} 3-\mathrm{N} 1$ & $127.4(4)$ & $\mathrm{C} 13-\mathrm{C} 18-\mathrm{C} 17$ & $120.8(4)$ \\
\hline $\mathrm{O} 1-\mathrm{C} 3-\mathrm{N} 1$ & $108.8(3)$ & $\mathrm{C} 13-\mathrm{C} 18-\mathrm{H} 18$ & 119.6 \\
\hline $\mathrm{C} 1-\mathrm{C} 4-\mathrm{H} 4 \mathrm{~A}$ & 109.5 & $\mathrm{C} 17-\mathrm{C} 18-\mathrm{H} 18$ & 119.6 \\
\hline $\mathrm{C} 1-\mathrm{C} 4-\mathrm{H} 4 \mathrm{~B}$ & 109.5 & $\mathrm{C} 12-\mathrm{C} 19-\mathrm{H} 19 \mathrm{~A}$ & 109.5 \\
\hline $\mathrm{H} 4 \mathrm{~A}-\mathrm{C} 4-\mathrm{H} 4 \mathrm{~B}$ & 109.5 & $\mathrm{C} 12-\mathrm{C} 19-\mathrm{H} 19 \mathrm{~B}$ & 109.5 \\
\hline $\mathrm{C} 1-\mathrm{C} 4-\mathrm{H} 4 \mathrm{C}$ & 109.5 & $\mathrm{H} 19 \mathrm{~A}-\mathrm{C} 19-\mathrm{H} 19 \mathrm{~B}$ & 109.5 \\
\hline $\mathrm{H} 4 \mathrm{~A}-\mathrm{C} 4-\mathrm{H} 4 \mathrm{C}$ & 109.5 & $\mathrm{C} 12-\mathrm{C} 19-\mathrm{H} 19 \mathrm{C}$ & 109.5 \\
\hline $\mathrm{H} 4 \mathrm{~B}-\mathrm{C} 4-\mathrm{H} 4 \mathrm{C}$ & 109.5 & $\mathrm{H} 19 \mathrm{~A}-\mathrm{C} 19-\mathrm{H} 19 \mathrm{C}$ & 109.5 \\
\hline $\mathrm{C} 6-\mathrm{C} 5-\mathrm{C} 10$ & $119.2(4)$ & $\mathrm{H} 19 \mathrm{~B}-\mathrm{C} 19-\mathrm{H} 19 \mathrm{C}$ & 109.5 \\
\hline $\mathrm{C} 6-\mathrm{C} 5-\mathrm{C} 2$ & $123.2(3)$ & $\mathrm{C} 16-\mathrm{C} 20-\mathrm{C} 21$ & $114.2(3)$ \\
\hline $\mathrm{C} 10-\mathrm{C} 5-\mathrm{C} 2$ & $117.6(4)$ & $\mathrm{C} 16-\mathrm{C} 20-\mathrm{H} 20 \mathrm{~A}$ & 108.7 \\
\hline $\mathrm{C} 5-\mathrm{C} 6-\mathrm{C} 7$ & $120.4(4)$ & $\mathrm{C} 21-\mathrm{C} 20-\mathrm{H} 20 \mathrm{~A}$ & 108.7 \\
\hline $\mathrm{C} 5-\mathrm{C} 6-\mathrm{H} 6$ & 119.8 & $\mathrm{C} 16-\mathrm{C} 20-\mathrm{H} 20 \mathrm{~B}$ & 108.7 \\
\hline $\mathrm{C} 7-\mathrm{C} 6-\mathrm{H} 6$ & 119.8 & $\mathrm{C} 21-\mathrm{C} 20-\mathrm{H} 20 \mathrm{~B}$ & 108.7 \\
\hline $\mathrm{C} 8-\mathrm{C} 7-\mathrm{C} 6$ & $120.1(4)$ & $\mathrm{H} 20 \mathrm{~A}-\mathrm{C} 20-\mathrm{H} 20 \mathrm{~B}$ & 107.6 \\
\hline $\mathrm{C} 8-\mathrm{C} 7-\mathrm{H} 7$ & 119.9 & $\mathrm{C} 22-\mathrm{C} 21-\mathrm{C} 23$ & $110.5(3)$ \\
\hline $\mathrm{C} 6-\mathrm{C} 7-\mathrm{H} 7$ & 119.9 & $\mathrm{C} 22-\mathrm{C} 21-\mathrm{C} 20$ & $110.6(4)$ \\
\hline $\mathrm{C} 7-\mathrm{C} 8-\mathrm{C} 9$ & $119.8(4)$ & $\mathrm{C} 23-\mathrm{C} 21-\mathrm{C} 20$ & $111.6(3)$ \\
\hline $\mathrm{C} 7-\mathrm{C} 8-\mathrm{H} 8$ & 120.1 & $\mathrm{C} 22-\mathrm{C} 21-\mathrm{H} 21$ & 108.0 \\
\hline $\mathrm{C} 9-\mathrm{C} 8-\mathrm{H} 8$ & 120.1 & $\mathrm{C} 23-\mathrm{C} 21-\mathrm{H} 21$ & 108.0 \\
\hline $\mathrm{C} 10-\mathrm{C} 9-\mathrm{C} 8$ & $120.1(4)$ & $\mathrm{C} 20-\mathrm{C} 21-\mathrm{H} 21$ & 108.0 \\
\hline $\mathrm{C} 10-\mathrm{C} 9-\mathrm{H} 9$ & 120.0 & $\mathrm{C} 21-\mathrm{C} 22-\mathrm{H} 22 \mathrm{~A}$ & 109.5 \\
\hline $\mathrm{C} 8-\mathrm{C} 9-\mathrm{H} 9$ & 120.0 & $\mathrm{C} 21-\mathrm{C} 22-\mathrm{H} 22 \mathrm{~B}$ & 109.5 \\
\hline $\mathrm{C} 9-\mathrm{C} 10-\mathrm{C} 5$ & $120.4(4)$ & $\mathrm{H} 22 \mathrm{~A}-\mathrm{C} 22-\mathrm{H} 22 \mathrm{~B}$ & 109.5 \\
\hline $\mathrm{C} 9-\mathrm{C} 10-\mathrm{H} 10$ & 119.8 & $\mathrm{C} 21-\mathrm{C} 22-\mathrm{H} 22 \mathrm{C}$ & 109.5 \\
\hline $\mathrm{C} 5-\mathrm{C} 10-\mathrm{H} 10$ & 119.8 & $\mathrm{H} 22 \mathrm{~A}-\mathrm{C} 22-\mathrm{H} 22 \mathrm{C}$ & 109.5 \\
\hline $\mathrm{O} 3-\mathrm{C} 11-\mathrm{N} 1$ & $118.3(3)$ & $\mathrm{H} 22 \mathrm{~B}-\mathrm{C} 22-\mathrm{H} 22 \mathrm{C}$ & 109.5 \\
\hline $\mathrm{O} 3-\mathrm{C} 11-\mathrm{C} 12$ & $121.1(3)$ & $\mathrm{C} 21-\mathrm{C} 23-\mathrm{H} 23 \mathrm{~A}$ & 109.5 \\
\hline $\mathrm{N} 1-\mathrm{C} 11-\mathrm{C} 12$ & $120.6(3)$ & $\mathrm{C} 21-\mathrm{C} 23-\mathrm{H} 23 \mathrm{~B}$ & 109.5 \\
\hline $\mathrm{C} 11-\mathrm{C} 12-\mathrm{C} 13$ & $110.5(3)$ & $\mathrm{H} 23 \mathrm{~A}-\mathrm{C} 23-\mathrm{H} 23 \mathrm{~B}$ & 109.5 \\
\hline $\mathrm{C} 11-\mathrm{C} 12-\mathrm{C} 19$ & $109.3(3)$ & $\mathrm{C} 21-\mathrm{C} 23-\mathrm{H} 23 \mathrm{C}$ & 109.5 \\
\hline $\mathrm{C} 13-\mathrm{C} 12-\mathrm{C} 19$ & $110.7(3)$ & $\mathrm{H} 23 \mathrm{~A}-\mathrm{C} 23-\mathrm{H} 23 \mathrm{C}$ & 109.5 \\
\hline $\mathrm{C} 11-\mathrm{C} 12-\mathrm{H} 12$ & 108.7 & $\mathrm{H} 23 \mathrm{~B}-\mathrm{C} 23-\mathrm{H} 23 \mathrm{C}$ & 109.5 \\
\hline $\mathrm{C} 13-\mathrm{C} 12-\mathrm{H} 12$ & 108.7 & $\mathrm{C} 11-\mathrm{N} 1-\mathrm{C} 3$ & $128.7(3)$ \\
\hline $\mathrm{C} 19-\mathrm{C} 12-\mathrm{H} 12$ & 108.7 & $\mathrm{C} 11-\mathrm{N} 1-\mathrm{C} 1$ & $120.9(3)$ \\
\hline $\mathrm{C} 18-\mathrm{C} 13-\mathrm{C} 14$ & $117.6(4)$ & $\mathrm{C} 3-\mathrm{N} 1-\mathrm{C} 1$ & $110.3(3)$ \\
\hline $\mathrm{C} 18-\mathrm{C} 13-\mathrm{C} 12$ & $119.2(3)$ & $\mathrm{C} 3-\mathrm{O} 1-\mathrm{C} 2$ & $109.5(3)$ \\
\hline $\mathrm{N} 1-\mathrm{C} 1-\mathrm{C} 2-\mathrm{O} 1$ & $27.6(3)$ & $\mathrm{C} 14-\mathrm{C} 15-\mathrm{C} 16-\mathrm{C} 17$ & $-0.7(6)$ \\
\hline $\mathrm{C} 4-\mathrm{C} 1-\mathrm{C} 2-\mathrm{O} 1$ & $-93.0(4)$ & $\mathrm{C} 14-\mathrm{C} 15-\mathrm{C} 16-\mathrm{C} 20$ & $179.9(3)$ \\
\hline $\mathrm{N} 1-\mathrm{C} 1-\mathrm{C} 2-\mathrm{C} 5$ & $149.7(3)$ & $\mathrm{C} 15-\mathrm{C} 16-\mathrm{C} 17-\mathrm{C} 18$ & $-0.2(6)$ \\
\hline $\mathrm{C} 4-\mathrm{C} 1-\mathrm{C} 2-\mathrm{C} 5$ & $29.1(5)$ & $\mathrm{C} 20-\mathrm{C} 16-\mathrm{C} 17-\mathrm{C} 18$ & $179.1(3)$ \\
\hline $\mathrm{O} 1-\mathrm{C} 2-\mathrm{C} 5-\mathrm{C} 6$ & $12.4(5)$ & $\mathrm{C} 14-\mathrm{C} 13-\mathrm{C} 18-\mathrm{C} 17$ & $0.0(6)$ \\
\hline $\mathrm{C} 1-\mathrm{C} 2-\mathrm{C} 5-\mathrm{C} 6$ & $-105.7(4)$ & $\mathrm{C} 12-\mathrm{C} 13-\mathrm{C} 18-\mathrm{C} 17$ & $174.4(3)$ \\
\hline $\mathrm{O} 1-\mathrm{C} 2-\mathrm{C} 5-\mathrm{C} 10$ & $-166.9(3)$ & $\mathrm{C} 16-\mathrm{C} 17-\mathrm{C} 18-\mathrm{C} 13$ & $0.6(6)$ \\
\hline
\end{tabular}




$\begin{array}{llll}\mathrm{C} 1-\mathrm{C} 2-\mathrm{C} 5-\mathrm{C} 10 & 75.0(4) & \mathrm{C} 17-\mathrm{C} 16-\mathrm{C} 20-\mathrm{C} 21 & -100.7(4) \\ \mathrm{C} 10-\mathrm{C} 5-\mathrm{C} 6-\mathrm{C} 7 & -1.3(6) & \mathrm{C} 15-\mathrm{C} 16-\mathrm{C} 20-\mathrm{C} 21 & 78.6(5) \\ \mathrm{C} 2-\mathrm{C} 5-\mathrm{C} 6-\mathrm{C} 7 & 179.4(4) & \mathrm{C} 16-\mathrm{C} 20-\mathrm{C} 21-\mathrm{C} 22 & -169.4(4) \\ \mathrm{C} 5-\mathrm{C} 6-\mathrm{C} 7-\mathrm{C} 8 & 0.8(7) & \mathrm{C} 16-\mathrm{C} 20-\mathrm{C} 21-\mathrm{C} 23 & 67.2(5) \\ \mathrm{C} 6-\mathrm{C} 7-\mathrm{C} 8-\mathrm{C} 9 & -0.6(7) & \mathrm{O} 3-\mathrm{C} 11-\mathrm{N} 1-\mathrm{C} 3 & -179.6(3) \\ \mathrm{C} 7-\mathrm{C} 8-\mathrm{C} 9-\mathrm{C} 10 & 1.1(6) & \mathrm{C} 12-\mathrm{C} 11-\mathrm{N} 1-\mathrm{C} 3 & 0.2(5) \\ \mathrm{C} 8-\mathrm{C} 9-\mathrm{C} 10-\mathrm{C} 5 & -1.6(6) & \mathrm{O} 3-\mathrm{C} 11-\mathrm{N} 1-\mathrm{C} 1 & 3.3(5) \\ \mathrm{C} 6-\mathrm{C} 5-\mathrm{C} 10-\mathrm{C} 9 & 1.8(6) & \mathrm{C} 12-\mathrm{C} 11-\mathrm{N} 1-\mathrm{C} 1 & -176.9(3) \\ \mathrm{C} 2-\mathrm{C} 5-\mathrm{C} 10-\mathrm{C} 9 & -179.0(4) & \mathrm{O} 2-\mathrm{C} 3-\mathrm{N} 1-\mathrm{C} 11 & 9.7(6) \\ \mathrm{O} 3-\mathrm{C} 11-\mathrm{C} 12-\mathrm{C} 13 & 89.6(4) & \mathrm{O} 1-\mathrm{C} 3-\mathrm{N} 1-\mathrm{C} 11 & -170.3(3) \\ \mathrm{N} 1-\mathrm{C} 11-\mathrm{C} 12-\mathrm{C} 13 & -90.2(4) & \mathrm{O} 2-\mathrm{C} 3-\mathrm{N} 1-\mathrm{C} 1 & -172.9(4) \\ \mathrm{O} 3-\mathrm{C} 11-\mathrm{C} 12-\mathrm{C} 19 & -32.5(4) & \mathrm{O} 1-\mathrm{C} 3-\mathrm{N} 1-\mathrm{C} 1 & 7.1(4) \\ \mathrm{N} 1-\mathrm{C} 11-\mathrm{C} 12-\mathrm{C} 19 & 147.7(3) & \mathrm{C} 4-\mathrm{C} 1-\mathrm{N} 1-\mathrm{C} 11 & -81.4(4) \\ \mathrm{C} 11-\mathrm{C} 12-\mathrm{C} 13-\mathrm{C} 18 & 146.6(4) & \mathrm{C} 2-\mathrm{C} 1-\mathrm{N} 1-\mathrm{C} 11 & 155.8(3) \\ \mathrm{C} 19-\mathrm{C} 12-\mathrm{C} 13-\mathrm{C} 18 & -92.1(4) & \mathrm{C} 4-\mathrm{C} 1-\mathrm{N} 1-\mathrm{C} 3 & 101.0(4) \\ \mathrm{C} 11-\mathrm{C} 12-\mathrm{C} 13-\mathrm{C} 14 & -39.3(5) & \mathrm{C} 2-\mathrm{C} 1-\mathrm{N} 1-\mathrm{C} 3 & -21.8(3) \\ \mathrm{C} 19-\mathrm{C} 12-\mathrm{C} 13-\mathrm{C} 14 & 82.0(4) & \mathrm{O} 2-\mathrm{C} 3-\mathrm{O} 1-\mathrm{C} 2 & -167.4(4) \\ \mathrm{C} 18-\mathrm{C} 13-\mathrm{C} 14-\mathrm{C} 15 & -0.9(6) & \mathrm{N} 1-\mathrm{C} 3-\mathrm{O} 1-\mathrm{C} 2 & 12.6(4) \\ \mathrm{C} 12-\mathrm{C} 13-\mathrm{C} 14-\mathrm{C} 15 & -175.1(3) & \mathrm{C} 5-\mathrm{C} 2-\mathrm{O} 1-\mathrm{C} 3 & -151.4(3) \\ \mathrm{C} 13-\mathrm{C} 14-\mathrm{C} 15-\mathrm{C} 16 & 1.3(6) & \mathrm{C} 1-\mathrm{C} 2-\mathrm{O} 1-\mathrm{C} 3 & -26.0(4) \\ \end{array}$

